K.-i. Watanabe and K. Yoshida

Nagoya Math. J.

Vol. 177 (2005), 47-75

\title{
HILBERT-KUNZ MULTIPLICITY OF THREE-DIMENSIONAL LOCAL RINGS
}

\author{
KEI-ICHI WATANABE AND KEN-ICHI YOSHIDA
}

\begin{abstract}
In this paper, we investigate the lower bound $s_{\mathrm{HK}}(p, d)$ of HilbertKunz multiplicities for non-regular unmixed local rings of Krull dimension $d$ containing a field of characteristic $p>0$. Especially, we focus on threedimensional local rings. In fact, as a main result, we will prove that $s_{\mathrm{HK}}(p, 3)=$ $4 / 3$ and that a three-dimensional complete local ring of Hilbert-Kunz multiplicity $4 / 3$ is isomorphic to the non-degenerate quadric hypersurface $k[[X, Y$, $Z, W]] /\left(X^{2}+Y^{2}+Z^{2}+W^{2}\right)$ under mild conditions.

Furthermore, we pose a generalization of the main theorem to the case of $\operatorname{dim} A \geq 4$ as a conjecture, and show that it is also true in case $\operatorname{dim} A=4$ using the similar method as in the proof of the main theorem.
\end{abstract}

\section{Introduction}

Let $A$ be a commutative Noetherian ring containing an infinite field of characteristic $p>0$ with unity. In [15], Kunz proved the following theorem, which gives a characterization of regular local rings of positive characteristic.

Kunz' Theorem. ([15]) Let $(A, \mathfrak{m}, k)$ be a local ring of characteristic $p>0$. Then the following conditions are equivalent:

(1) A is a regular local ring.

(2) $A$ is reduced and is flat over the subring $A^{p}=\left\{a^{p}: a \in A\right\}$. In other words, the Frobenius map $F: A \rightarrow A\left(a \mapsto a^{p}\right)$ is flat.

(3) $l_{A}\left(A / \mathfrak{m}^{[q]}\right)=q^{d}$ for any $q=p^{e}, e \geq 1$, where $\mathfrak{m}^{[q]}=\left(a^{q}: a \in \mathfrak{m}\right)$ and $l_{A}(M)$ denotes the length of an A-module $M$.

Received August 25, 2003.

2000 Mathematics Subject Classification: Primary 13D40, 13A35; Secondary 13H05, $13 \mathrm{H} 10,13 \mathrm{H} 15$.

The first author was supported in part by Grant aid in Scientific Researches, \#13440015 and \#13874006.

The second author was supported in part by NSF Grant \#14540020. 
Furthermore, in [16], Kunz observed that $l_{A}\left(A / \mathfrak{m}^{[q]}\right) / q^{d}\left(q=p^{e}\right)$ is a reasonable measure for the singularity of a local ring. Based on the idea of Kunz, Monsky [18] proved that there exists a constant $c=c(A)$ such that

$$
l_{A}\left(A / \mathfrak{m}^{[q]}\right)=c q^{d}+O\left(q^{d-1}\right)
$$

and defined the notion of Hilbert-Kunz multiplicity by $e_{\mathrm{HK}}(A)=c$. In 1990's, Han and Monsky [10] have given an algorism to compute the HilbertKunz multiplicity for any hypersurface of Briskorn-Fermat type

$$
A=k\left[X_{0}, \ldots, X_{n}\right] /\left(X_{0}^{d_{0}}+\cdots+X_{n}^{d_{n}}\right) .
$$

See e.g. [1], [2], [4], [24] about the other examples. Hochster and Huneke [11] have given a "Length Criterion for Tight Closure" in terms of Hilbert-Kunz multiplicity (see Theorem 1.8) and indicated the close relation between tight closure and Hilbert-Kunz multiplicity. In [22], the authors proved a theorem which gives a characterization of regular local rings in terms of Hilbert-Kunz multiplicity:

Theorem A. ([22, Theorem 1.5]) Let $(A, \mathfrak{m}, k)$ be an unmixed local ring of positive characteristic. Then $A$ is regular if and only if $e_{\mathrm{HK}}(A)=1$.

Many researchers have tried to improve this theorem. For example, Blickle and Enescu [3] recently proved the following theorem:

Theorem B. (Blickle-Enescu [3]) Let $(A, \mathfrak{m}, k)$ be an unmixed local ring of characteristic $p>0$. Then the following statements hold:

(1) If $e_{\mathrm{HK}}(A)<1+\frac{1}{d !}$, then $A$ is Cohen-Macaulay and F-rational.

(2) If $e_{\mathrm{HK}}(A)<1+\frac{1}{p^{d} d !}$, then $A$ is regular.

So it is natural to consider the following problem:

Problem C. Let $d \geq 2$ be any integer. Determine the lower bound $\left(s_{\mathrm{HK}}(p, d)\right)$ of Hilbert-Kunz multiplicities for d-dimensional non-regular unmixed local rings of characteristic $p$. Also, characterize the local rings $A$ for which $e_{\mathrm{HK}}(A)=s_{\mathrm{HK}}(p, d)$ holds.

In case of one-dimensional local rings, it is easy to answer to this problem. In fact, $s_{\mathrm{HK}}(p, 1)=2 ; e_{\mathrm{HK}}(A)=2$ if and only if $e(A)=2$. In case of two-dimensional Cohen-Macaulay local rings, the authors [23] have given a complete answer to this problem. Namely, we have $s_{\mathrm{HK}}(p, 2)=\frac{3}{2}$ by the theorem below. 
Theorem D. (see also Corollary 2.6) Let $(A, \mathfrak{m}, k)$ be a two-dimensional Cohen-Macaulay local ring of positive characteristic. Put $e=e(A)$, the multiplicity of $A$. Then the following statements hold:

(1) $e_{\mathrm{HK}}(A) \geq \frac{e+1}{2}$.

(2) Suppose that $k=\bar{k}$. Then $e_{\mathrm{HK}}(A)=\frac{e+1}{2}$ holds if and only if the associated graded ring $\operatorname{gr}_{\mathfrak{m}}(A)$ is isomorphic to the Veronese subring $k[X, Y]^{(e)}$.

In the following, let us explain the organization of this paper. In Section 1 , we recall the notions of Hilbert-Kunz multiplicity and tight closure etc. and gather several fundamental properties of them. In particular, GotoNakamura's theorem (Theorem 1.9) is important because it plays a central role in the proof of the main result (Theorem 3.1).

In Section 2, we give a key result to estimate Hilbert-Kunz multiplicities for local rings of lower dimension. Indeed, Theorem 2.2 is a refinement of the argument in [23, Section 2]. Also, the point of our proof is to estimate $l_{A}\left(\mathfrak{m}{ }^{[q]} / J^{[q]}\right.$ ) (where $J$ is a minimal reduction of $\mathfrak{m}$ ) using volumes in $\mathbb{R}^{d}$.

In Section 3, we prove the following theorem as the main result in this paper.

Theorem 3.1. Let $(A, \mathfrak{m}, k)$ be a three-dimensional unmixed local ring of characteristic $p>0$. Then the following statements hold.

(1) If $A$ is not regular, then $e_{\mathrm{HK}}(A) \geq \frac{4}{3}$.

(2) Suppose that $k=\bar{k}$ and char $k \neq 2$. Then the following conditions are equivalent:

(a) $e_{\mathrm{HK}}(A)=\frac{4}{3}$.

(b) $\widehat{A} \cong k[[X, Y, Z, W]] /\left(X^{2}+Y^{2}+Z^{2}+W^{2}\right)$.

Also, we study lower bounds on $e_{\mathrm{HK}}(A)$ for local rings $A$ having a given (small) multiplicity $e$. In particular, we will prove that any three-dimensional unmixed local ring $A$ with $e_{\mathrm{HK}}(A)<2$ is $F$-rational.

In Section 4, we consider a generalization of Theorem 3.1 and pose the following conjecture:

Conjecture 4.2. Let $d \geq 1$ be an integer and $p>2$ a prime number. Put

$$
A_{p, d}:=\overline{\mathbb{F}_{p}}\left[\left[X_{0}, X_{1}, \ldots, X_{d}\right]\right] /\left(X_{0}^{2}+\cdots+X_{d}^{2}\right) .
$$


Let $(A, \mathfrak{m}, k)$ be a d-dimensional unmixed local ring with $k=\overline{\mathbb{F}_{p}}$. Then the following statements hold.

(1) If $A$ is not regular, then $e_{\mathrm{HK}}(A) \geq e_{\mathrm{HK}}\left(A_{p, d}\right) \geq 1+\frac{c_{d}}{d !}$ (see 4.2 for the definition of $\left.c_{d}\right)$. In particular, $s_{\mathrm{HK}}(p, d)=e_{\mathrm{HK}}\left(A_{p, d}\right)$.

(2) If $e_{\mathrm{HK}}(A)=e_{\mathrm{HK}}\left(A_{p, d}\right)$, then the $\mathfrak{m}$-adic completion $\widehat{A}$ of $A$ is isomorphic to $A_{p, d}$ as local rings.

Also, we prove that this is true in case of $\operatorname{dim} A=4$. Namely we will prove the following theorem.

ThEOREM 4.3. Let $(A, \mathfrak{m}, k)$ be a four-dimensional unmixed local ring of characteristic $p>0$. Also, suppose that $k=\bar{k}$ and char $k \neq 2$. Then $e_{\mathrm{HK}}(A) \geq \frac{5}{4}$ if $e(A) \geq 3$. Also, the following statements hold.

(1) If $A$ is not regular, then $e_{\mathrm{HK}}(A) \geq e_{\mathrm{HK}}\left(A_{p, 4}\right)=\frac{29 p^{2}+15}{24 p^{2}+12}$.

(2) The following conditions are equivalent:

(a) Equality holds in (1).

(b) $e_{\mathrm{HK}}(A)<\frac{5}{4}$.

(c) $\widehat{A}$ is isomorphic to $A_{p, 4}$.

\section{$\S 1$. Preliminaries}

Throughout this paper, let $A$ be a commutative Noetherian ring with unity. Furthermore, we assume that $A$ has a positive characteristic $p$, that is, it contains a prime field $\mathbb{F}_{p}=\mathbb{Z} / p \mathbb{Z}$, unless otherwise specified. For every positive integer $e$, let $q=p^{e}$. If $I$ is an ideal of $A$, then $I^{[q]}=\left(a^{q}: a \in I\right) A$. Also, we fix the following notation: $l_{A}(M)\left(\operatorname{resp} . \mu_{A}(M)\right)$ denotes the length (resp. the minimal number of generators) of $M$ for any finitely generated $A$-module $M$.

First, we recall the notion of Hilbert-Kunz multiplicity (see [15], [16], [18]). Also, see [17] or [20] for usual multiplicity.

Definition 1.1. (multiplicity, Hilbert-Kunz multiplicity) Let $(A, \mathfrak{m}, k)$ be a local ring of characteristic $p>0$ with $\operatorname{dim} A=d$. Let $I$ be an $\mathfrak{m}$ primary ideal of $A$, and let $M$ be a finitely generated $A$-module. The (Hilbert-Samuel) multiplicity $e(I, M)$ of $I$ with respect to $M$ is defined by

$$
e(I, M)=\lim _{n \rightarrow \infty} \frac{d !}{n^{d}} l_{A}\left(M / I^{n} M\right) .
$$


The Hilbert-Kunz multiplicity $e_{\mathrm{HK}}(I, M)$ of $I$ with respect to $M$ is defined by

$$
e_{\mathrm{HK}}(I, M)=\lim _{q \rightarrow \infty} \frac{l_{A}\left(M / I^{[q]} M\right)}{q^{d}} .
$$

By definition, we put $e(I)=e(I, A)\left(\operatorname{resp} . e_{\mathrm{HK}}(I)=e_{\mathrm{HK}}(I, A)\right)$ and $e(A)=$ $e(\mathfrak{m})\left(\operatorname{resp} . e_{\mathrm{HK}}(A)=e_{\mathrm{HK}}(\mathfrak{m})\right)$.

We recall several basic results on Hilbert-Kunz multiplicity.

Proposition 1.2. (Fundamental properties (cf. [13], [15], [16], [18], $[22]))$ Let $(A, \mathfrak{m}, k)$ be a local ring of positive characteristic. Let $I, I^{\prime}$ be $\mathfrak{m}$-primary ideals of $A$, and let $M$ be a finitely generated $A$-module. Then the following statements hold.

(1) If $I \subseteq I^{\prime}$, then $e_{\mathrm{HK}}(I) \geq e_{\mathrm{HK}}\left(I^{\prime}\right)$.

(2) $e_{\mathrm{HK}}(A) \geq 1$.

(3) $\operatorname{dim} M<d$ if and only if $e_{\mathrm{HK}}(I, M)=0$.

(4) If $0 \rightarrow L \rightarrow M \rightarrow N \rightarrow 0$ is a short exact sequence of finitely generated $A$-modules, then

$$
e_{\mathrm{HK}}(I, M)=e_{\mathrm{HK}}(I, L)+e_{\mathrm{HK}}(I, N) .
$$

(5) (Associative formula)

$$
e_{\mathrm{HK}}(I, M)=\sum_{\mathfrak{p} \in \operatorname{Assh}(A)} e_{\mathrm{HK}}(I, A / \mathfrak{p}) \cdot l_{A_{\mathfrak{p}}}\left(M_{\mathfrak{p}}\right),
$$

where $\operatorname{Assh}(A)$ denotes the set of prime ideals $\mathfrak{p}$ of $A$ with $\operatorname{dim} A / \mathfrak{p}=$ $\operatorname{dim} A$.

(6) If $J$ is a parameter ideal of $A$, then $e_{\mathrm{HK}}(J)=e(J)$. In particular, if $J$ is a minimal reduction of $I$ (i.e., $J$ is a parameter ideal which is contained in $I$ and $I^{r+1}=J I^{r}$ for some integer $r \geq 0$ ), then $e_{\mathrm{HK}}(J)=e(I)$.

(7) If $A$ is regular, then $e_{\mathrm{HK}}(I)=l_{A}(A / I)$.

(8) (Localization) $e_{\mathrm{HK}}\left(A_{\mathfrak{p}}\right) \leq e_{\mathrm{HK}}(A)$ holds for any prime ideal $\mathfrak{p}$ such that $\operatorname{dim} A / \mathfrak{p}+$ height $\mathfrak{p}=\operatorname{dim} A$.

(9) If $x \in I$ is A-regular, then $e_{\mathrm{HK}}(I) \leq e_{\mathrm{HK}}(I / x A)$. 
(10) If $(A, \mathfrak{m}) \rightarrow(B, \mathfrak{n})$ is a flat local ring homomorphism such that $B / \mathfrak{m} B$ is a field, then $e_{\mathrm{HK}}(I)=e_{\mathrm{HK}}(I B)$.

Remark 1. Also, the similar result as above (except (6), (7)) holds for usual multiplicities.

Let $(A, \mathfrak{m}, k)$ be any local ring of positive dimension. The associated graded ring $\operatorname{gr}_{\mathfrak{m}}(A)$ of $A$ with respect to $\mathfrak{m}$ is defined as follows:

$$
\operatorname{gr}_{\mathfrak{m}}(A):=\bigoplus_{n \geq 0} \mathfrak{m}^{n} / \mathfrak{m}^{n+1}
$$

Then $G=\operatorname{gr}_{\mathfrak{m}}(A)$ is a homogeneous $k$-algebra such that $\mathfrak{M}:=G_{+}$is the unique homogeneous maximal ideal of $G$. If $\operatorname{char} A=p>0$ and $\operatorname{dim} A=d$, then $G_{\mathfrak{M}}$ is also a local ring of characteristic $p$ with $\operatorname{dim} G_{\mathfrak{M}}=d$.

Proposition 1.3. ([22, Theorem $(2.15)])$ Let $(A, \mathfrak{m}, k)$ be a local ring of positive characteristic. Let $G=\operatorname{gr}_{\mathfrak{m}}(A)$ the associated graded ring of $A$ with respect $\mathfrak{m}$ as above. Then $e_{\mathrm{HK}}(A) \leq e_{\mathrm{HK}}\left(G_{\mathfrak{M}}\right) \leq e(A)$.

Remark 2. We use the same notation as in the above proposition. Although $e(A)=e\left(G_{\mathfrak{M}}\right)$ always holds, $e_{\mathrm{HK}}(A)=e_{\mathrm{HK}}\left(G_{\mathfrak{M}}\right)$ seldom holds.

Proposition 1.4. (cf. [13]) Let $(A, \mathfrak{m}, k)$ be a local ring of positive characteristic with $d=\operatorname{dim} A$. Let $I$ be an $\mathfrak{m}$-primary ideal of $A$. Then

$$
\frac{e(I)}{d !} \leq e_{\mathrm{HK}}(I) \leq e(I)
$$

Also, if $d \geq 2$, then the inequality in the left-hand side is strict; see [9].

We say that a local $\operatorname{ring} A$ is unmixed if $\operatorname{dim} \widehat{A} / \mathfrak{p}=\operatorname{dim} \widehat{A}$ holds for any associated prime ideal $\mathfrak{p}$ of $\widehat{A}$. The following theorem is an analogy of Nagata's theorem $([20,(40.6)])$, which is a starting point in this article.

ThEOREm 1.5. ([22, Theorem (1.5)]) Let $(A, \mathfrak{m}, k)$ be an unmixed local ring of positive characteristic. Then $A$ is regular if and only if $e_{\mathrm{HK}}(A)=1$.

It is not so easy to compute Hilbert-Kunz multiplicities in general. However, one has simple formulas for them in case of quotient singularities and in case of binomial hypersurfaces; see below or [4, Theorem 3.1]. 
Theorem 1.6. (cf. [22, Theorem (2.7)]) Let $(A, \mathfrak{m}) \hookrightarrow(B, \mathfrak{n})$ be a module-finite extension of local domains of positive characteristic. Then for every $\mathfrak{m}$-primary ideal $I$ of $A$, we have

$$
e_{\mathrm{HK}}(I)=\frac{e_{\mathrm{HK}}(I B)}{[Q(B): Q(A)]} \cdot[B / \mathfrak{n}: A / \mathfrak{m}],
$$

where $Q(A)$ denotes the fraction field of $A$.

Now let us see some examples of Hilbert-Kunz multiplicities which are given by the above formula. First, we consider the Veronese subring $A$ defined by

$$
A=k\left[\left[X_{1}^{i_{1}} \cdots X_{d}^{i_{d}}: i_{1}, \ldots, i_{d} \geq 0, \sum i_{j}=r\right]\right] .
$$

Applying Theorem 1.6 to $A \hookrightarrow B=k\left[\left[X_{1}, \ldots, X_{d}\right]\right]$, we get

$$
e_{\mathrm{HK}}(A)=\frac{1}{r}\left(\begin{array}{c}
d+r-1 \\
r-1
\end{array}\right) \text {. }
$$

In particular, if $d=2, r=e(A)$, then $e_{\mathrm{HK}}(A)=\frac{e(A)+1}{2}$.

Next, we consider the homogeneous coordinate rings of quadric hypersurfaces in $\mathbb{P}_{k}^{3}$. Let $k$ be a field of characteristic $p>2$, and let $R$ be the homogeneous coordinate ring of the hyperquadric $Q$ defined by $q=q(X, Y, Z, W)$. Put $\mathfrak{M}=R_{+}$, the unique homogeneous maximal ideal of $R$, and $A=R_{\mathfrak{M}} \otimes_{k} \bar{k}$. By suitable coordinate transformation, we may assume that $\widehat{A}$ is isomorphic to one of the following rings:

$$
\begin{cases}k[[X, Y, Z, W]] /\left(X^{2}\right), & \text { if } \operatorname{rank}(q)=1, \\ k[[X, Y, Z, W]] /\left(X^{2}-Y Z\right), & \text { if } \operatorname{rank}(q)=2, \\ k[[X, Y, Z, W]] /(X Y-Z W), & \text { if } \operatorname{rank}(q)=3 .\end{cases}
$$

Then $e_{\mathrm{HK}}(A)=2, \frac{3}{2}$, or $\frac{4}{3}$, respectively.

In order to state other important properties of Hilbert-Kunz multiplicity, the notion of tight closure is very important. See [11], [12], [13] for definition and the fundamental properties of tight closure. In particular, the notion of $F$-rational ring is essential in our argument.

Definition 1.7. ([6], [11], [12]) Let $(A, \mathfrak{m}, k)$ be a local ring of positive characteristic. We say that $A$ is weakly $F$-regular (resp. $F$-rational) if every ideal (resp. every parameter ideal) is tightly closed. Also, $A$ is $F$ regular (resp. F-rational) if any local ring of $A$ is weakly $F$-regular (resp. $F$-rational). 
Note that an F-rational local ring is normal and Cohen-Macaulay.

Hochster and Huneke have given the following criterion of tight closure in terms of Hilbert-Kunz multiplicity.

Theorem 1.8. (Length Criterion for Tight Closure (cf. [11, Theorem 8.17])) Let $I \subseteq J$ be $\mathfrak{m}$-primary ideals of a local ring $(A, \mathfrak{m}, k)$ of positive characteristic.

(1) If $I^{*}=J^{*}$, then $e_{\mathrm{HK}}(I)=e_{\mathrm{HK}}(J)$.

(2) Suppose that $A$ is excellent, reduced and equidimensional. Then the converse of (1) is also true.

The following theorem plays an important role in studying HilbertKunz multiplicities for non-Cohen-Macaulay local rings.

Theorem 1.9. (Goto-Nakamura [8]) Let $(A, \mathfrak{m}, k)$ be an equidimensional local ring which is a homomorphic image of a Cohen-Macaulay local ring of characteristic $p>0$. Then

(1) If $J$ is a parameter ideal of $A$, then $e(J) \geq l_{A}\left(A / J^{*}\right)$.

(2) Suppose that $A$ is unmixed. If $e(J)=l_{A}\left(A / J^{*}\right)$ for some parameter ideal $J$, then $A$ is F-rational (hence is Cohen-Macaulay).

The next corollary is well-known in case of Cohen-Macaulay local rings (e.g. see $[13])$.

Corollary 1.10. Let $(A, \mathfrak{m}, k)$ be an unmixed local ring of characteristic $p>0$. Suppose that $e(A)=2$. Then $\widehat{A}$ is F-rational if and only if $e_{\mathrm{HK}}(A)<2$. When this is the case, $A$ is an $F$-rational hypersurface.

Proof. Since any Cohen-Macaulay local ring of multiplicity 2 is a hypersurface, it suffices to prove the first statement.

We may assume that $A$ is complete and $k$ is infinite. We can take a minimal reduction $J$ of $\mathfrak{m}$. First, suppose that $e_{\mathrm{HK}}(A)<2$. Then we show that $A$ is Cohen-Macaulay, $F$-rational. By Goto-Nakamura's theorem, we have $2=e(J) \geq l_{A}\left(A / J^{*}\right)$. If equality does not hold, then $l_{A}\left(A / J^{*}\right)=1$, that is, $J^{*}=\mathfrak{m}$. Then $e_{\mathrm{HK}}(A)=e_{\mathrm{HK}}\left(J^{*}\right)=e_{\mathrm{HK}}(J)=e(J)=2$ by Proposition 1.2. This is a contradiction. Hence $e(J)=l_{A}\left(A / J^{*}\right)$. By Goto-Nakamura's theorem again, we obtain that $A$ is Cohen-Macaulay, $F$ rational. 
Conversely, suppose that $A$ is a complete $F$-rational local ring. Then since $A$ is Cohen-Macaulay and $J^{*}=J \neq \mathfrak{m}$, we have $e_{\mathrm{HK}}(A)<e_{\mathrm{HK}}(J)=$ $e(J)=2$ by the Length Criterion for Tight Closure.

The next question is open in general. However, we will show that it is true for $\operatorname{dim} A \leq 3$; see Section 3 .

Question 1.11. If $A$ is an unmixed local ring with $e_{\mathrm{HK}}(A)<2$, then is it F-rational?

\section{§2. Estimate of Hilbert-Kunz multiplicities}

In this section, we will prove the key result to find a lower bound on Hilbert-Kunz multiplicities. Actually, it is a refinement of the argument which appeared in [22, Section 5] or in [23, Section 2]. The point is to use the tight closure $J^{*}$ instead of "a parameter ideal $J$ itself". This enables us to investigate Hilbert-Kunz multiplicities of non-Cohen-Macaulay local rings. In Sections 3, 4, we will apply our method to unmixed local rings with $\operatorname{dim} A=3,4$.

Before stating our theorem, we introduce the following notation: Fix $d>0$. For any positive real number $s$, we put

$$
v_{s}:=\operatorname{vol}\left\{\left(x_{1}, \ldots, x_{d}\right) \in[0,1]^{d}: \sum_{i=1}^{d} x_{i} \leq s\right\}, \quad v_{s}^{\prime}:=1-v_{s}
$$

where $\operatorname{vol}(W)$ denotes the volume of $W \subseteq \mathbb{R}^{d}$. Then it is easy to see the following fact.

FACT 2.1. Let s be a positive real number. Using the same notation as above, we have

(1) $v_{s}+v_{s}^{\prime}=1$

(2) $v_{d-s}^{\prime}=v_{s}$.

(3) $v_{d / 2}=v_{d / 2}^{\prime}=\frac{1}{2}$.

(4) If $0 \leq s \leq 1$, then $v_{s}=\frac{s^{d}}{d !}$.

Using the above notaion, the key result in this paper can be written as follows: 
THEOREM 2.2. Let $(A, \mathfrak{m}, k)$ be an unmixed local ring of characteristic $p>0$. Put $d=\operatorname{dim} A \geq 1$. Let $J$ be a minimal reduction of $\mathfrak{m}$, and let $r$ be an integer with $r \geq \mu_{A}\left(\mathfrak{m} / J^{*}\right)$, where $J^{*}$ denotes the tight closure of $J$. Also, let $s \geq 1$ be a rational number. Then we have

$$
e_{\mathrm{HK}}(A) \geq e(A)\left\{v_{s}-r \cdot \frac{(s-1)^{d}}{d !}\right\} \text {. }
$$

Remark 3. When $1 \leq s \leq 2$, the right-hand side in Equation (2.1) is equal to $e(A)\left(v_{s}-r \cdot v_{s-1}\right)$.

Before proving the theorem, we need the following lemma. In what follows, for any positive real number $\alpha$, we define $I^{\alpha}:=I^{n}$, where $n$ is the minimum integer which does not exceed $\alpha$.

LEMMA 2.3. Let $(A, \mathfrak{m}, k)$ be an unmixed local ring of characteristic $p>0$ with $\operatorname{dim} A=d \geq 1$. Let $J$ be a parameter ideal of $A$. Using the same notation as above, we have

$$
\lim _{q \rightarrow \infty} \frac{l_{A}\left(A / J^{s q}\right)}{q^{d}}=\frac{e(J) s^{d}}{d !}, \quad \lim _{q \rightarrow \infty} l_{A}\left(\frac{J^{s q}+J^{[q]}}{J[q]}\right)=e(J) \cdot v_{s}^{\prime} .
$$

Proof. First, note that our assertion holds if $A$ is regular and $J=\mathfrak{m}$. We may assume that $A$ is complete. Let $x_{1}, \ldots, x_{d}$ be a system of parameters which generates $J$, and put $R:=k\left[\left[x_{1}, \ldots, x_{d}\right]\right], \mathfrak{n}=\left(x_{1}, \ldots, x_{d}\right) R$. Then $R$ is a complete regular local ring and $A$ is a finitely generated $R$ module with $A / \mathfrak{m}=R / \mathfrak{n}$. Since the assertion is clear in case of regular local rings, it suffices to show the following claim.

Claim. Let $\mathcal{I}=\left\{I_{q}\right\}_{q=p^{e}}$ be a set of ideals of $A$ which satisfies the following conditions:

(1) For each $q=p^{e}, I_{q}=J_{q} A$ holds for some ideal $J_{q} \subseteq R$.

(2) There exists a positive integer $t$ such that $\mathfrak{n}^{t q} \subseteq J_{q}$ for all $q=p^{e}$.

(3) $\lim _{q \rightarrow \infty} l_{R}\left(R / J_{q}\right) / q^{d}$ exists.

Then

$$
\lim _{q \rightarrow \infty} \frac{l_{A}\left(A / I_{q}\right)}{q^{d}}=e(J) \cdot \lim _{q \rightarrow \infty} \frac{l_{R}\left(R / J_{q}\right)}{q^{d}} .
$$


In fact, since $A$ is unmixed, it is a torsion-free $R$-module of rank $e:=$ $e(J)$. Take a free $R$-module $F$ of rank $e$ such that $A_{W} \cong F_{W}$, where $W=R \backslash\{0\}$. Since $F$ and $A$ are both torsion-free, there exist the following short exact sequences of finitely generated $R$-modules:

$$
0 \rightarrow F \rightarrow A \rightarrow C_{1} \rightarrow 0, \quad 0 \rightarrow A \rightarrow F \rightarrow C_{2} \rightarrow 0,
$$

where $\left(C_{1}\right)_{W}=\left(C_{2}\right)_{W}=0$. In particular, $\operatorname{dim} C_{1}<d$ and $\operatorname{dim} C_{2}<d$.

Applying the tensor product $-\otimes_{R} R / J_{q}$ to the above two exact sequences, respectively, we get

$$
\begin{aligned}
l_{A}\left(A / I_{q}\right) & \leq l_{R}\left(F / J_{q} F\right)+l_{R}\left(C_{1} / J_{q} C_{1}\right), \\
l_{R}\left(F / J_{q} F\right) & \leq l_{A}\left(A / I_{q}\right)+l_{R}\left(C_{2} / J_{q} C_{2}\right) .
\end{aligned}
$$

In general, if $\operatorname{dim}_{R} C<d$, then

$$
\frac{l_{R}\left(C / J_{q} C\right)}{q^{d}} \leq \frac{l_{R}\left(C / \mathfrak{n}^{t q} C\right)}{q^{d}} \rightarrow 0 \quad(q \rightarrow \infty) .
$$

Thus the required assertion easily follows from the above observation.

Proof of Theorem 2.2. For simplicity, we put $L=J^{*}$ and $e=e(A)$. We will give an upper bound of $l_{A}\left(\mathfrak{m}^{[q]} / J^{[q]}\right)$. First, we have the following inequality:

$$
\begin{aligned}
l_{A}\left(\mathfrak{m}^{[q]} / J^{[q]}\right) \leq & l_{A}\left(\frac{\mathfrak{m}^{[q]}+\mathfrak{m}^{s q}}{J^{[q]}}\right) \\
= & l_{A}\left(\frac{\mathfrak{m}^{[q]}+\mathfrak{m}^{s q}}{L^{[q]}+\mathfrak{m}^{s q}}\right)+l_{A}\left(\frac{L^{[q]}+\mathfrak{m}^{s q}}{L^{[q]}+J^{s q}}\right) \\
& \quad+l_{A}\left(\frac{L^{[q]}+J^{s q}}{J^{[q]}+J^{s q}}\right)+l_{A}\left(\frac{J^{[q]}+J^{s q}}{J^{[q]}}\right) \\
= & : \ell_{1}+\ell_{2}+\ell_{3}+\ell_{4} .
\end{aligned}
$$

Next, we see that $\ell_{1} \leq r \cdot l_{A}\left(A / J^{(s-1) q}\right)+O\left(q^{d-1}\right)$. By our assumption, we can write $\mathfrak{m}=L+A a_{1}+\cdots+A a_{r}$. Since $\mathfrak{m}^{(s-1) q} a_{i}^{q} \subseteq \mathfrak{m}^{s q} \subseteq \mathfrak{m}^{s q}+L^{[q]}$, we have

$$
\begin{aligned}
\ell_{1}=l_{A}\left(\frac{\mathfrak{m}^{[q]}+\mathfrak{m}^{s q}}{L^{[q]}+\mathfrak{m}^{s q}}\right) & \leq \sum_{i=1}^{r} l_{A}\left(\frac{A a_{i}^{q}+L^{[q]}+\mathfrak{m}^{s q}}{L^{[q]}+\mathfrak{m}^{s q}}\right) \\
& =\sum_{i=1}^{r} l_{A}\left(A /\left(L^{[q]}+\mathfrak{m}^{s q}\right): a_{i}^{q}\right) \\
& \leq r \cdot l_{A}\left(A / \mathfrak{m}^{(s-1) q}\right) .
\end{aligned}
$$


Since $J$ is a minimal reduction of $\mathfrak{m}$, we have $l_{A}\left(\mathfrak{m}^{(s-1) q} / J^{(s-1) q}\right)=O\left(q^{d-1}\right)$. Thus we have the required inequality. Similarly, we get

$$
\ell_{2}=l_{A}\left(\frac{L^{[q]}+\mathfrak{m}^{s q}}{L^{[q]}+J^{s q}}\right) \leq l_{A}\left(\mathfrak{m}^{s q} / J^{s q}\right)=O\left(q^{d-1}\right)
$$

Also, we have $l_{A}\left(L^{[q]} / J^{[q]}\right)=O\left(q^{d-1}\right)$ by Length Criterion for Tight Closure. Hence $\ell_{3}=O\left(q^{d-1}\right)$ and thus

$$
l_{A}\left(\mathfrak{m}^{[q]} / J^{[q]}\right) \leq r \cdot l_{A}\left(A / J^{(s-1) q}\right)+l_{A}\left(\frac{J^{[q]}+J^{s q}}{J^{[q]}}\right)+O\left(q^{d-1}\right) .
$$

It follows from the above argument that

$$
\begin{aligned}
e_{\mathrm{HK}}(J)-e_{\mathrm{HK}}(\mathfrak{m}) & \leq r \cdot \lim _{q \rightarrow \infty} \frac{l_{A}\left(A / J^{(s-1) q}\right)}{q^{d}}+\lim _{q \rightarrow \infty} \frac{1}{q^{d}} l_{A}\left(\frac{J^{[q]}+J^{s q}}{J^{[q]}}\right) \\
& =r \cdot e \cdot \frac{(s-1)^{d}}{d !}+e \cdot v_{s}^{\prime} .
\end{aligned}
$$

Since $e_{\mathrm{HK}}(J)=e(J)=e, e_{\mathrm{HK}}(A)=e_{\mathrm{HK}}(\mathfrak{m})$ and $v_{s}^{\prime}=1-v_{s}$, we get the required inequality.

The following fact is known, which gives a lower bound on Hilbert-Kunz multiplicities for hypersurface local rings.

FACT 2.4. (cf. [1], [2], [22]) Let $(A, \mathfrak{m}, k)$ be a hypersurface local ring of characteristic $p>0$ with $d=\operatorname{dim} A \geq 1$. Then

$$
e_{\mathrm{HK}}(A) \geq \beta_{d+1} \cdot e(A)
$$

where $\beta_{d+1}$ is given by the following equivalent formulas:

$$
\begin{aligned}
& \text { (a) } \frac{1}{\pi} \int_{-\infty}^{\infty}\left(\frac{\sin \theta}{\theta}\right)^{d+1} d \theta \\
& \text { (b) } \frac{1}{2^{d} d !} \sum_{\ell=0}^{\left[\frac{d}{2}\right]}(-1)^{\ell}(d+1-2 \ell)^{d}\left(\begin{array}{c}
d+1 \\
\ell
\end{array}\right) ; \\
& \text { (c) } \operatorname{vol}\left\{\underline{x} \in[0,1]^{d}: \frac{d-1}{2} \leq \sum x_{i} \leq \frac{d+1}{2}\right\}=1-v_{\frac{d-1}{2}}-v_{\frac{d+1}{2}}^{\prime} .
\end{aligned}
$$


TABLE 1.

\begin{tabular}{|c||c|c|c|c|c|c|c|}
\hline$d$ & 0 & 1 & 2 & 3 & 4 & 5 & 6 \\
\hline$\beta_{d+1}$ & 1 & 1 & $\frac{3}{4}$ & $\frac{2}{3}$ & $\frac{115}{192}$ & $\frac{11}{20}$ & $\frac{5633}{11520}$ \\
\hline
\end{tabular}

Remark 4. The above inequality is not best possible in general. In case of $d \geq 4$, one cannot prove the formula in the above fact as a corollary of our theorem. See also Proposition 3.9 and Theorem 4.3.

The following lemma is an analogy of Sally's theorem: If $A$ is a CohenMacaulay local ring, then $\mu_{A}(\mathfrak{m} / J)=\mu_{A}(\mathfrak{m})-\operatorname{dim} A \leq e(A)-1$.

LEMma 2.5. Let $(A, \mathfrak{m}, k)$ be an unmixed local ring of positive characteristic, and let $J$ be a minimal reduction of $\mathfrak{m}$.

(1) $\mu_{A}\left(\mathfrak{m} / J^{*}\right) \leq e(A)-1$.

(2) If $A$ is not $F$-rational, then $\mu_{A}\left(\mathfrak{m} / J^{*}\right) \leq e(A)-2$.

Proof. We may assume that $A$ is complete and thus is a homomorphic image of a Cohen-Macaulay local ring.

(1) By Goto-Nakamura's Theorem, we have that $\mu_{A}\left(\mathfrak{m} / J^{*}\right) \leq l_{A}\left(\mathfrak{m} / J^{*}\right)$ $\leq e(J)-1=e-1$.

(2) If $A$ is not $F$-rational, then $l_{A}\left(A / J^{*}\right) \leq e(J)-1=e-1$. Thus $\mu_{A}\left(\mathfrak{m} / J^{*}\right) \leq e-2$, as required.

Using Theorem 2.2 and Lemma 2.5, one can prove the following corollary, which has been already proved in [23] in the case of Cohen-Macaulay local rings.

Corollary 2.6. (cf. [23]) Let $(A, \mathfrak{m}, k)$ be a two-dimensional unmixed local ring of characteristic $p>0$. Put $e=e(A)$. Then

$$
e_{\mathrm{HK}}(A) \geq \frac{e+1}{2} \text {. }
$$

Also, suppose $k=\bar{k}$. Then the equality holds if and only if $\operatorname{gr}_{\mathfrak{m}}(A)$ is isomorphic to the Veronese subring $k[X, Y]^{(e)}=k\left[X^{e}, X^{e-1} Y, \ldots, X Y^{e-1}, Y^{e}\right]$.

Moreover, if $A$ is not $F$-rational, then we have

$$
e_{\mathrm{HK}}(A) \geq \frac{e^{2}}{2(e-1)} .
$$


Example 2.7. (Fakhruddin-Trivedi [7, Corollary 3.19]) Let $E$ be an elliptic curve over a field $k=\bar{k}$ of characteristic $p>0$, and let $\mathcal{L}$ be a very ample line bundle on $E$ of degree $e \geq 2$. Let $R$ be the homogeneous coordinate ring (the section ring of $\mathcal{L}$ ) defined by

$$
R=\bigoplus_{n \geq 0} H^{0}\left(E, \mathcal{L}^{\otimes n}\right) .
$$

Also, put $A=R_{\mathfrak{M}}$, where $\mathfrak{M}$ be the unique homogeneous maximal ideal of $R$. Then we have $e_{\mathrm{HK}}(A)=\frac{e^{2}}{2(e-1)}$.

\section{§3. Lower bounds in the case of three-dimensional local rings}

In this section, we prove the main theorem in this paper, which gives the lower bound of Hilbert-Kunz multiplicities for non-regular unmixed local rings of dimension 3 .

TheOREm 3.1. Let $(A, \mathfrak{m}, k)$ be a three-dimensional unmixed local ring of characteristic $p>0$. Then

(1) If $A$ is not regular, then $e_{\mathrm{HK}}(A) \geq \frac{4}{3}$.

(2) Suppose that $k=\bar{k}$ and char $k \neq 2$. Then the following conditions are equivalent:

(a) $e_{\mathrm{HK}}(A)=\frac{4}{3}$.

(b) $\widehat{A} \cong k[[X, Y, Z, W]] /\left(X^{2}+Y^{2}+Z^{2}+W^{2}\right)$.

(c) $\operatorname{gr}_{\mathfrak{m}}(A) \cong k[X, Y, Z, W] /\left(X^{2}+Y^{2}+Z^{2}+W^{2}\right)$. That is, $\operatorname{gr}_{\mathfrak{m}}(A) \cong$ $k[X, Y, Z, W] /(X Y-Z W)$.

Proposition 3.2. Let $(A, \mathfrak{m}, k)$ be a three-dimensional unmixed local ring of characteristic $p>0$. If $e_{\mathrm{HK}}(A)<2$, then $A$ is $F$-rational.

From now on, we divide the proof of Theorem 3.1 and Proposition 3.2 into several steps. In the following, we assume the following condition.

$(\#)$ : Let $(A, \mathfrak{m}, k)$ be a three-dimensional unmixed local ring of characteristic $p>0$, and $e(A)=e$, the multiplicity of $A$. Also, suppose that $\mathfrak{m}$ has a minimal reduction $J$.

Suppose that $A$ is not regular under the assumption (\#). Then $e=$ $e(A)$ is an integer with $e \geq 2$. Thus the first assertion of Theorem 3.1 follows from the following lemma. Also, this implies that if $e_{\mathrm{HK}}(A)=\frac{4}{3}$ then $e(A)=2$ without extra assumptions. 
LEMMA 3.3. Under the assumption (\#), we have

(1) If $e \geq 5$, then $e_{\mathrm{HK}}(A)>2$.

(2) If $e=4$, then $e_{\mathrm{HK}}(A) \geq \frac{7}{4}>\frac{4}{3}$.

(3) If $e=3$, then $e_{\mathrm{HK}}(A) \geq \frac{13}{8}>\frac{4}{3}$.

(4) If $e=2$, then $e_{\mathrm{HK}}(A) \geq \frac{4}{3}$.

Remark 5. The lower bounds of $e_{\mathrm{HK}}(A)$ in Lemma 3.3 are not best possible.

Proof. We may assume that $A$ is complete. By Lemma 2.5(1), we can apply Theorem 2.2 with $r=e-1$. Namely, if $1 \leq s \leq 2$, then

$$
e_{\mathrm{HK}}(A) \geq e\left(v_{s}-(e-1) v_{s-1}\right)=e\left(\frac{s^{3}}{6}-(e+2) \frac{(s-1)^{3}}{6}\right) \text {. }
$$

Define the real-valued function $f_{e}(s)$ by the right-hand side of Eq. (3.1). Then one can easily calculate $\max _{1 \leq s \leq 2} f_{e}(s)$. In fact, if $e \geq 2$, then

$$
\max _{1 \leq s \leq 2} f_{e}(s)=f\left(\frac{e+2+\sqrt{e+2}}{e+1}\right)=\frac{e}{6}\left(\frac{e+2+\sqrt{e+2}}{e+1}\right)^{2} .
$$

But, in order to prove the lemma, it is enough to use the following values only:

\begin{tabular}{|c||c|c|c|}
\hline$s$ & $\frac{3}{2}$ & $\frac{7}{4}$ & 2 \\
\hline$f_{e}(s)$ & $\frac{e(25-e)}{48}$ & $\frac{e(289-27 e)}{384}$ & $\frac{e(6-e)}{6}$ \\
\hline
\end{tabular}

(1) We show that $e_{\mathrm{HK}}(A)>2$ if $e \geq 5$. If $e \geq 13$, then by Proposition 1.4,

$$
e_{\mathrm{HK}}(A) \geq \frac{e}{3 !} \geq \frac{13}{6}>2
$$

So we may assume that $5 \leq e \leq 12$. Applying Eq. (3.1) for $s=\frac{3}{2}$, we get

$$
e_{\mathrm{HK}}(A) \geq \frac{e(25-e)}{48} \geq \frac{5(25-5)}{48}=\frac{25}{12}>2 \text {. }
$$

(2) Suppose that $e=4$. Applying Eq. (3.1) for $s=\frac{3}{2}$, we get

$$
e_{\mathrm{HK}}(A) \geq \frac{e(25-e)}{48}=\frac{7}{4}
$$


(3) Suppose that $e=3$. Applying Eq. (3.1) for $s=\frac{7}{4}$, we get

$$
e_{\mathrm{HK}}(A) \geq \frac{e(289-27 e)}{384}=\frac{13}{8} \text {. }
$$

(4) Suppose that $e=2$. Applying Eq. (3.1) for $s=2$,

$$
e_{\mathrm{HK}}(A) \geq \frac{e(6-e)}{6}=\frac{4}{3}
$$

as required.

Before proving the second assertion of Theorem 3.1, we prove Proposition 3.2. For that purpose, we now focus non- $F$-rational local rings.

Now suppose that $A$ is not $F$-rational. If $e=2$, then $e_{\mathrm{HK}}(A)=2$ by Lemma 1.10. On the other hand, if $e \geq 5$, then $e_{\mathrm{HK}}(A)>2$ by Lemma 3.3. Thus in order to prove Proposition 3.2, it is enough to investigate the cases of $e=3,4$. Namely, Proposition 3.2 follows from the following lemma.

Lemma 3.4. Suppose that $A$ is not F-rational under the assumption (\#). Then

(1) If $e=3$, then $e_{\mathrm{HK}}(A) \geq 2$.

(2) If $e=4$, then $e_{\mathrm{HK}}(A)>2$.

Proof. By Lemma 2.5(2), we can apply Theorem 2.2 for $r=e-2$. Thus if $1 \leq s \leq 2$, then

$$
e_{\mathrm{HK}}(A) \geq e\left(\frac{s^{3}}{6}-(e+1) \frac{(s-1)^{3}}{6}\right) .
$$

(1) Suppose that $e=3$. Applying Eq. (3.2) for $s=2$, we get

$$
e_{\mathrm{HK}}(A) \geq \frac{e(7-e)}{6}=2 \text {. }
$$

(2) Suppose that $e=4$. Applying Eq. (3.2) for $s=\frac{7}{4}$, we get

$$
e_{\mathrm{HK}}(A) \geq \frac{e(316-27 e)}{384}=\frac{13}{6}>2,
$$

as required. 
EXAMPLE 3.5. Let $R=k\left[T, x T, x y T, y T, x^{-1} y T, x^{-2} y T, \ldots, x^{-n} y T\right]$ be a rational normal scroll and put $\mathfrak{m}=\left(T, x T, x y T, y T, x^{-1} y T, \ldots, x^{-n} y T\right)$. Then $A=R_{\mathfrak{m}}$ is a three-dimensional Cohen-Macaulay $F$-rational local domain with $e(A)=n+2$, and

$$
e_{\mathrm{HK}}(A)=\frac{e(A)}{2}+\frac{e(A)}{6(n+1)} .
$$

Proof. Let $\mathcal{P} \subseteq \mathbb{R}$ be a convex polytope with vertex set

$$
\Gamma=\{(0,0),(1,0),(1,1),(0,1),(-1,1), \ldots,(-n, 1)\},
$$

and put $\widetilde{\mathcal{P}}:=\left\{(\alpha, 1) \in \mathbb{R}^{3}: \alpha \in \mathcal{P}\right\}$ and $d \mathcal{P}:=\{d \cdot \alpha: \alpha \in \mathcal{P}\}$ for every integer $d \geq 0$. Also, if we define a cone $\mathcal{C}=\mathcal{C}(\widetilde{\mathcal{P}}):=\{r \beta: \beta \in \widetilde{\mathcal{P}}, 0 \leq r \in$ $\mathbb{Q}\}$ and regard $R$ as a homogeneous $k$-algebra with $\operatorname{deg} x=\operatorname{deg} y=0$ and $\operatorname{deg} T=1$, then the basis of $R_{d}$ corresponds to the set $\left\{(\alpha, d) \in \mathbb{Z}^{3}: \alpha \in\right.$ $\left.\mathbb{Z}^{2} \cap d \mathcal{P}\right\}=\left\{(\alpha, d) \in \mathbb{Z}^{3}: \alpha \in \mathbb{Z}^{2}\right\} \cap \mathcal{C}$.

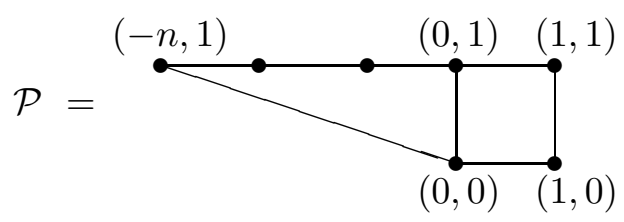

If we put $\Gamma_{q}=\{(0,0),(q, 0),(q, q),(0, q),(-q, q), \ldots,(-n q, q)\}$, then $\mathfrak{m}^{[q]}=\left(x^{a} y^{b} T^{q}:(a, b) \in \Gamma_{q}\right)$. Since $\left[\mathfrak{m}^{[q]}\right]_{d}=\sum_{(a, b) \in \Gamma_{q}} R_{d-q} x^{a} y^{b} T^{q}$, we have

$$
\begin{aligned}
e_{\mathrm{HK}}(A) & =\lim _{q \rightarrow \infty} \frac{1}{q^{3}} l_{A}\left(A / \mathfrak{m}^{[q]}\right) \\
& =\lim _{q \rightarrow \infty} \frac{1}{q^{3}} \#\left\{\mathbb{Z}^{3} \cap\left(\mathcal{C} \backslash \bigcup_{(a, b) \in \Gamma_{q}}(a, b, q)+\mathcal{C}\right)\right\}
\end{aligned}
$$

that is,

$$
e_{\mathrm{HK}}(A)=\lim _{q \rightarrow \infty} \frac{1}{q^{3}}\left[\sum_{d=0}^{\infty} \#\left\{\mathbb{Z}^{2} \cap\left(d \mathcal{P} \backslash \bigcup_{(a, b) \in \Gamma_{q}}(a, b)+\max \{0, d-q\} \mathcal{P}\right)\right\}\right] .
$$

Also, if we define a real continuous function $f:[0, \infty) \rightarrow \mathbb{R}$ by

$$
f(t)=\text { the volume of }\left[t \mathcal{P} \backslash \bigcup_{(a, b) \in \Gamma}(a, b)+\max \{0, t-1\} \mathcal{P}\right] \text { in } \mathbb{R}^{2},
$$


then $e_{\mathrm{HK}}(A)=\int_{0}^{\infty} f(t) d t$. Let us denote the volume of $M \subseteq \mathbb{R}^{2}$ by $\operatorname{vol}(M)$. To calculate $e_{\mathrm{HK}}(A)$, we need to determine $f(t)$. Namely, we need to show the following claim.

Claim.

$$
f(t)= \begin{cases}\operatorname{vol}(t \mathcal{P}), & 0 \leq t<1 \\ \operatorname{vol}(t \mathcal{P})-(n+4) \operatorname{vol}((t-1) \mathcal{P}), & 1 \leq t<\frac{n+2}{n+1} \\ \frac{(n+2) t(2-t)}{2}+(n+2) \frac{(2-t)^{2}}{2 n}, & \frac{n+2}{n+1} \leq t<2 \\ 0, & t \geq 2\end{cases}
$$

To prove the claim, we may assume that $t \geq 1$. For simplicity, we put $M_{a, b}=(a, b)+(t-1) \mathcal{P}$ for every $(a, b) \in \Gamma$. First suppose that $1 \leq t<\frac{n+2}{n+1}$. Then since $1-n(t-1)>t-1, M_{0,0} \cap M_{1,0}=\emptyset$. Similarly, one can easily see that any two $M_{a, b}$ do not intersect each other; see Figure 1. Thus $f(t)=\operatorname{vol}(t \mathcal{P})-(n+4) \operatorname{vol}((t-1) \mathcal{P})$.

Next suppose that $\frac{n+2}{n+1} \leq t<2$. Then $\mathcal{P} \cap\left\{(x, y) \in \mathbb{R}^{2}: 0 \leq y \leq\right.$ $t-1\}=M_{0,0} \cup M_{1,0} \cup T_{0}$, where $T_{0}$ is a triangle with vertex $(t-1,0),(1,0)$ and $\left(t-1, \frac{2-t}{n}\right)$. Similarly, there exist $(n+1)$-triangles $T_{1}, \ldots, T_{n+1}$ having the same volumes as $T_{0}$ such that

$\mathcal{P} \cap\left\{(x, y) \in \mathbb{R}^{2}: 1 \leq y \leq t\right\}=M_{-n, 1} \cup \cdots \cup M_{1,1} \cup M_{0,1} \cup M_{1,1} \cup T_{1} \cup \cdots \cup T_{n+1}$

and any two $T_{i}$ 's do not intersect each other; see Figure 2. Thus

$$
\begin{aligned}
f(t) & =\operatorname{vol}\left(\mathcal{P} \cap\left\{(x, y) \in \mathbb{R}^{2}: t-1 \leq y \leq 1\right\}\right)+(n+2) \operatorname{vol}\left(T_{0}\right) \\
& =\frac{(n+2) t(2-t)}{2}+(n+2) \frac{(2-t)^{2}}{2 n} .
\end{aligned}
$$

Finally, suppose that $t \geq 2$. Then since $\mathcal{P}$ is covered by $M_{a, b}$ 's, we have $f(t)=0$, as required.

Using the above claim, let us calculate $e_{\mathrm{HK}}(A)$. Note that $\operatorname{vol}(t \mathcal{P})=$ $\frac{(n+2) t^{2}}{2}$.

$$
\begin{aligned}
e_{\mathrm{HK}}(A)= & \int_{0}^{\frac{n+2}{n+1}} \frac{(n+2) t^{2}}{2} d t-(n+4) \int_{1}^{\frac{n+2}{n+1}} \frac{(n+2)(t-1)^{2}}{2} d t \\
& +\int_{\frac{n+2}{n+1}}^{2} \frac{(n+2) t(2-t)}{2} d t+(n+2) \int_{\frac{n+2}{n+1}}^{2} \frac{(2-t)^{2}}{2 n} d t \\
= & (n+2)\left[\frac{1}{2}+\frac{1}{6(n+1)}\right]
\end{aligned}
$$




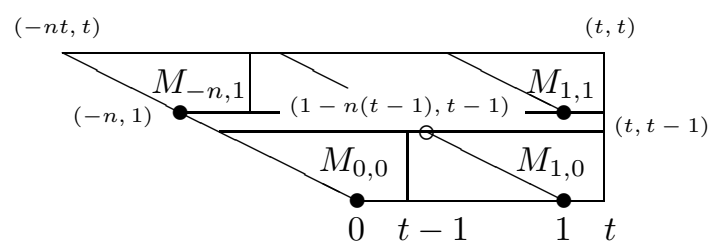

Figure 1. The case where $1 \leq t<\frac{n+2}{n+1}$

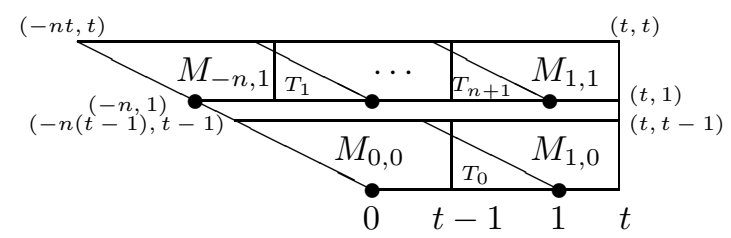

Figure 2. The case where $\frac{n+2}{n+1} \leq t<2$

as required.

Discussion 3.6. Let $A$ be a complete local ring which satisfies (\#). Also, suppose that $e=3$. What is the smallest value of $e_{\mathrm{HK}}(A)$ among such rings?

The function $f_{e}(s)=3\left(\frac{s^{3}}{6}-5 \frac{(s-1)^{3}}{6}\right)$, which appeared in Eq. (3.1), takes the maximal value

$$
f\left(\frac{5+\sqrt{5}}{4}\right)=\frac{15+5 \sqrt{5}}{16}=1.636 \cdots
$$

in $s \in[1,2]$. Hence $e_{\mathrm{HK}}(A) \geq 1.636 \cdots$. But we believe that this is not best possible.

Suppose that $e_{\mathrm{HK}}(A)<2$. Then $A$ is $F$-rational by Lemma 3.4. Thus it is Cohen-Macaulay and $3+1 \leq v=\operatorname{emb}(A) \leq d+e-1=3+3-1=5$. If $v \neq 5$, then $A$ is a hypersurface and $e_{\mathrm{HK}}(A) \geq \frac{2}{3} \cdot e=2$ by Fact 2.4 . Hence we may assume that $v=5$, that is, $A$ has maximal embedding dimension. If we write as $A=R / I$, where $R$ is a complete regular local ring with $\operatorname{dim} R=5$, then height $I=2$. By Hilbert-Burch's theorem, there exists a $2 \times 3$-matrix $\mathbb{M}$ such that $I=I_{2}(\mathbb{M})$, the ideal generated by all 2-minors of M. In particular, $A$ can be written as $A=B / a B$, where $B=k[X] / I_{2}(X)$, 
$X$ is a generic $2 \times 3$-matrix and $a$ is a prime element of $B$. This implies that

$$
e_{\mathrm{HK}}(A)=e_{\mathrm{HK}}(B / a B) \geq e_{\mathrm{HK}}(B)=3\left\{\frac{1}{2}+\frac{1}{4 !}\right\}=\frac{13}{8}=1.625 ;
$$

see $[5$, Section 3].

For example, if $A=k\left[\left[T, x T, x y t, y T, x^{-1} y T\right]\right]$ is a rational normal scroll, then $e_{\mathrm{HK}}(A)=\frac{7}{4}=1.75$ by Example 3.5 . Is this the smallest value?

Discussion 3.7. Let $A$ be a complete local ring which satisfies (\#). Also, suppose that $e=4$. What is the smallest value of $e_{\mathrm{HK}}(A)$ among such rings?

As in Discussion 3.6, it suffices to consider $F$-rational local rings only. For example, let $A=k[[x, y, z]]^{(2)}$ be the Veronese subring. Then $A$ is an $F$-rational local domain with $e(A)=4$ and $e_{\mathrm{HK}}(A)=2$. Also, let $A$ be the completion of the Rees algebra $R(\mathfrak{n})$ over an $F$-rational double point $(R, \mathfrak{n})$ of dimension 2. Then $A$ is an $F$-rational local domain with $e(A)=4$ and $e_{\mathrm{HK}}(A) \geq 2$ (we believe that this inequality is strict).

On the other hand, the function $f_{e}(s)$ which appeared in Eq. (3.1), takes the maximal value

$$
f\left(\frac{6+\sqrt{6}}{5}\right)=\frac{28+8 \sqrt{6}}{25}=1.903 \cdots
$$

in $s \in[1,2]$. Hence the fact that we can prove now is " $e_{\mathrm{HK}}(A) \geq 1.903 \ldots$ " only.

Based on Corollary 2.6 and Discussion 3.7, we pose the following conjecture.

Conjecture 3.8. Let $A$ be a complete local ring which satisfies (\#), and let $r \geq 2$ be an integer. If $e(A)=r^{2}$, then

$$
e_{\mathrm{HK}}(A) \geq \frac{(r+1)(r+2)}{6} \text {. }
$$

Also, the equality holds if and only if $A$ is isomorphic to $k[[x, y, z]]^{(r)}$.

In the rest of this section, we prove the second statement of Theorem 3.1. Let $(A, \mathfrak{m}, k)$ be a complete local ring which satisfies (\#). If 
$e_{\mathrm{HK}}(A)=\frac{4}{3}$, then $A$ is an $F$-rational hypersurface with $e(A)=2$ by the above observation. Furthermore, suppose that $k=\bar{k}$ and char $k \neq 2$. Then we may assume that $A$ can be written as the form $k[[X, Y, Z, W]] /\left(X^{2}-\right.$ $\varphi(Y, Z, W))$. To study Hilbert-Kunz multiplicities for these rings, we prove the improved version of Theorem 2.2.

Proposition 3.9. Let $k$ be an algebraically closed field of char $k \neq 2$, and let $A=k[[X, Y, Z, W]] /\left(X^{2}-\varphi(Y, Z, W)\right)$ be an $F$-rational hypersurface local ring. Let $a, b, c$ be integers with $2 \leq a \leq b \leq c$.

Suppose that there exists a function ord $: A \rightarrow \mathbb{Q} \cup\{\infty\}$ which satisfies the following conditions:

(1) $\operatorname{ord}(\alpha) \geq 0 ;$ and $\operatorname{ord}(\alpha)=\infty \Longleftrightarrow \alpha=0$.

(2) $\operatorname{ord}(x)=1 / 2, \operatorname{ord} y=1 / a$, ord $z=1 / b$, and ord $w=1 / c$.

(3) $\operatorname{ord}(\varphi) \geq 1$.

(4) $\operatorname{ord}(\alpha+\beta) \geq \min \{\operatorname{ord}(\alpha), \operatorname{ord}(\beta)\}$.

(5) $\operatorname{ord}(\alpha \beta) \geq \operatorname{ord}(\alpha)+\operatorname{ord}(\beta)$.

Then we have

$$
e_{\mathrm{HK}}(A) \geq 2-\frac{a b c}{12}\left(N^{3}-n^{3}\right)
$$

where

$$
N=\frac{1}{a}+\frac{1}{b}+\frac{1}{c}-\frac{1}{2}, \quad n=\max \left\{0, N-\frac{2}{c}\right\} .
$$

In particular, if $(a, b, c) \neq(2,2,2)$, then $e_{\mathrm{HK}}(A)>\frac{4}{3}$.

Remark 6. The third condition ord $(\varphi) \geq 1$ is important. For example, if $\varphi \equiv y^{2} \bmod (z, w)^{3}$, then one can take $(a, b, c)=(2,3,3)$, but $(a, b, c)=$ $(2,3,4)$.

Proof. First, we define a filtration $\left\{F_{n}\right\}_{n \in \mathbb{Q}}$ as follows:

$$
F_{n}:=\{\alpha \in A: \operatorname{ord}(\alpha) \geq n\}
$$

Then every $F_{n}$ is an ideal and $F_{m} F_{n} \subseteq F_{m+n}$ holds for all $m, n \in \mathbb{Q}$. Using $F_{n}$ instead of $\mathfrak{m}^{n}$, we shall estimate $l_{A}\left(\mathfrak{m}^{[q]} / J^{[q]}\right)$.

Set $J=(y, z, w) A$ and fix a sufficiently large power $q=p^{e}$. Put

$$
s=\frac{1}{a}+\frac{1}{b}+\frac{1}{c}, \quad N=\frac{1}{a}+\frac{1}{b}+\frac{1}{c}-\frac{1}{2} .
$$


Since $J$ is a minimal reduction of $\mathfrak{m}$ and $x y^{q-1} z^{q-1} w^{q-1}$ generates the socle of $A / J^{[q]}$, we have that $F_{s q} \subseteq J^{[q]}$. Also, since $B=A / J^{[q]}$ is an Artinian Gorenstein local ring, we get

$$
F_{\frac{(N+1) q}{2}} B \subseteq 0:_{B} F_{\frac{N q}{2}} B \cong K_{B / F_{\frac{N q}{2}} B}
$$

where $K_{C}$ denotes a canonical module of a local ring $C$. Hence, by the Matlis duality theorem, we get

$$
l_{A}\left(\frac{F_{\frac{(N+1) q}{2}}+J^{[q]}}{J^{[q]}}\right) \leq l_{B}\left(F_{\frac{(N+1) q}{2}}\right) \leq l_{B}\left(K_{B / F_{\frac{N q}{2}} B}\right)=l_{B}\left(B / F_{\frac{N q}{2}} B\right) .
$$

On the other hand, since $x^{q} \in F_{\frac{q}{2}}$ by the assumption, we have

$$
x^{q} F_{\frac{N q}{2}} \subseteq F_{\frac{(N+1) q}{2}} .
$$

Therefore by a similar argument as in the proof of Theorem 2.2, we get

$$
\begin{aligned}
& l_{A}\left(\mathfrak{m}^{[q]} / J^{[q]}\right) \leq l_{A}\left(\frac{A x^{q}+J^{[q]}+F_{\frac{(N+1) q}{2}}}{F_{\frac{(N+1) q}{2}}+J[q]}\right)+l_{A}\left(\frac{\frac{F_{(N+1) q}}{2}+J^{[q]}}{J[q]}\right) \\
& \leq l_{A}\left(A /\left(J^{[q]}+F_{\frac{(N+1) q}{2}}\right): x^{q}\right)+l_{B}\left(B / F_{\frac{N q}{2}} B\right) \\
& \leq 2 \cdot l_{A}\left(A / J^{[q]}+F_{\frac{N}{2} q}\right) \text {. }
\end{aligned}
$$

In fact, since

$$
\begin{aligned}
\lim _{q \rightarrow \infty} & \frac{1}{q^{3}} l_{A}\left(A / J^{[q]}+F_{\frac{N q}{2}}\right) \\
& =e(A) \cdot \lim _{q \rightarrow \infty} \frac{1}{q^{3}} \operatorname{vol}\left\{(x, y, z) \in[0, q]^{3}: \frac{y}{a}+\frac{z}{b}+\frac{w}{c} \leq \frac{N q}{2}\right\} \\
& =2 \cdot \operatorname{vol}\left\{(x, y, z) \in[0,1]^{3}: \frac{y}{a}+\frac{z}{b}+\frac{w}{c} \leq \frac{N}{2}\right\} \\
& =\frac{a b c}{24}\left(N^{3}-n^{3}\right)
\end{aligned}
$$

we get

$$
e_{\mathrm{HK}}(A) \geq 2-2 \cdot \frac{a b c}{24}\left(N^{3}-n^{3}\right)=2-\frac{a b c}{12}\left(N^{3}-n^{3}\right),
$$

as required. 
EXAMPLE 3.10. Let $k$ be an algebraically closed field of char $k \neq 2$, and let $(A, \mathfrak{m}, k)$ be a hypersurface. Put $\operatorname{gr}_{\mathfrak{m}}(A)=k[X, Y, Z, W] /(g(X, Y$, $Z, W))$.

$$
\begin{aligned}
& g(X, Y, Z, W)=X^{2}+Y^{3}+Z^{3}+W^{3} \Longrightarrow e_{\mathrm{HK}}(A) \geq \frac{55}{32} \\
& g(X, Y, Z, W)=X^{2}+Y^{2}+Z^{3}+W^{3} \Longrightarrow e_{\mathrm{HK}}(A) \geq \frac{14}{9} \\
& g(X, Y, Z, W)=X^{2}+Y^{2}+Z^{2}+W^{c} \Longrightarrow e_{\mathrm{HK}}(A) \geq \frac{3}{2}-\frac{2}{3 c^{2}} .
\end{aligned}
$$

Proof of Theorem 3.1(2). Put $G=\operatorname{gr}_{\mathfrak{m}}(A)$ and $\mathfrak{M}=\operatorname{gr}_{\mathfrak{m}}(A)_{+}$. The implication $(a) \Rightarrow(b)$ follows from Proposition 3.9. $(b) \Rightarrow(c)$ is clear. Suppose (c). Then $e_{\mathrm{HK}}\left(G_{\mathfrak{M}}\right)=\frac{4}{3}$. Also, by Proposition 1.3 and Theorem 3.1(1), we have that $\frac{4}{3} \leq e_{\mathrm{HK}}(A) \leq e_{\mathrm{HK}}\left(G_{\mathfrak{M}}\right)=\frac{4}{3}$. Thus $e_{\mathrm{HK}}(A)=\frac{4}{3}$, as required.

Also, the following corollary follows from the proof of Proposition 3.9 and Example 3.10.

Corollary 3.11. Let $A$ be a local ring which satisfies (\#). Also, assume that $k=\bar{k}$ and $p \neq 2$. Then the following conditions are equivalent:

(1) $\frac{4}{3}<e_{\mathrm{HK}}(A) \leq \frac{3}{2}$.

(2) $\operatorname{gr}_{\mathfrak{m}}(A) \cong k[X, Y, Z] /\left(X^{2}+Y^{2}+Z^{2}\right)$.

(3) $A$ is isomorphic to a hypersurface $k[[X, Y, Z, W]] /\left(X^{2}+Y^{2}+Z^{2}+W^{c}\right)$ for some integer $c \geq 3$.

When this is the case, $e_{\mathrm{HK}}(A) \geq \frac{3}{2}-\frac{2}{3 c^{2}}$.

\section{§4. A generalization of the main result to higher dimensional case}

In this section, we want to generalize Theorem 3.1 to the case of $\operatorname{dim} A \geq 4$. Let $d \geq 1$ be an integer and $p>2$ a prime number. If we put

$$
A_{p, d}:=\overline{\mathbb{F}_{p}}\left[\left[X_{0}, X_{1}, \ldots, X_{d}\right]\right] /\left(X_{0}^{2}+\cdots+X_{d}^{2}\right),
$$

then we can guess that $e_{\mathrm{HK}}\left(A_{p, d}\right)=s_{\mathrm{HK}}(p, d)$ holds according to the observations until the previous section. In the following, let us formulate this as a conjecture and prove that it is also true in case of $\operatorname{dim} A=4$. 
In [10], Han and Monsky gave an algorism to calculate $e_{\mathrm{HK}}\left(A_{p, d}\right)$, but it is not so easy to represent $e_{\mathrm{HK}}\left(A_{p, d}\right)$ as a quotient of two polynomials of $p$ for any fixed $d \geq 1$.

\begin{tabular}{|c||c|c|c|c|}
\hline$d$ & 1 & 2 & 3 & 4 \\
\hline$e_{\mathrm{HK}}\left(A_{p, d}\right)$ & 2 & $\frac{3}{2}$ & $\frac{4}{3}$ & $\frac{29 p^{2}+15}{24 p^{2}+12}$ \\
\hline
\end{tabular}

On the other hand, surprisingly, Monsky proved the following theorem:

THEOREM 4.1. (Monsky [19]) Under the above notation, we have

$$
\lim _{p \rightarrow \infty} e_{\mathrm{HK}}\left(A_{p, d}\right)=1+\frac{c_{d}}{d !}
$$

where

$$
\sec x+\tan x=\sum_{d=0}^{\infty} \frac{c_{d}}{d !} x^{d} \quad\left(|x|<\frac{\pi}{2}\right) .
$$

Remark 7. It is known that the Taylor expansion of sec $x($ resp. $\tan x)$ at origin can be written as follows:

$$
\begin{aligned}
\sec x & =\sum_{i=0}^{\infty} \frac{E_{2 i}}{(2 i) !} x^{2 i}, \\
\tan x & =\sum_{i=1}^{\infty}(-1)^{i-1} \frac{2^{2 i}\left(2^{2 i}-1\right) B_{2 i}}{(2 i) !} x^{2 i-1},
\end{aligned}
$$

where $E_{2 i}$ (resp. $B_{2 i}$ ) is said to be Euler number (resp. Bernoulli number).

Also, $c_{d}$ appeared in Eq. (4.1) is a positive integer since cos $t$ is an unit element in a ring $\mathcal{H}=\left\{\sum_{n=0}^{\infty} a_{n} \frac{t^{n}}{n !}: a_{n} \in \mathbb{Z}\right.$ for all $\left.n \geq 0\right\}$.

Based on the above observation, we pose the following conjecture.

CONJECTURE 4.2. Let $d \geq 1$ be an integer and $p>2$ a prime number. Put

$$
A_{p, d}:=\overline{\mathbb{F}_{p}}\left[\left[X_{0}, X_{1}, \ldots, X_{d}\right]\right] /\left(X_{0}^{2}+\cdots+X_{d}^{2}\right) .
$$

Let $(A, \mathfrak{m}, k)$ be a d-dimensional unmixed local ring with $k=\overline{\mathbb{F}_{p}}$. Then the following statements hold.

(1) If $A$ is not regular, then $e_{\mathrm{HK}}(A) \geq e_{\mathrm{HK}}\left(A_{p, d}\right) \geq 1+\frac{c_{d}}{d !}$. In particular, $s_{\mathrm{HK}}(p, d)=e_{\mathrm{HK}}\left(A_{p, d}\right)$. 
(2) If $e_{\mathrm{HK}}(A)=e_{\mathrm{HK}}\left(A_{p, d}\right)$, then $\widehat{A} \cong A_{p, d}$ as local rings.

In the following, we prove that this is true in case of $\operatorname{dim} A=4$. Note that

$$
\lim _{p \rightarrow \infty} e_{\mathrm{HK}}\left(A_{p, 4}\right)=\lim _{p \rightarrow \infty} \frac{29 p^{2}+15}{24 p^{2}+12}=\frac{29}{24}=1+\frac{c_{4}}{4 !} .
$$

THEOREM 4.3. Let $(A, \mathfrak{m}, k)$ be an unmixed local ring of characteristic $p>0$ with $\operatorname{dim} A=4$. If $e(A) \geq 3$, then $e_{\mathrm{HK}}(A) \geq \frac{5}{4}=\frac{30}{24}$.

Suppose that $k=\bar{k}$ and char $k \neq 2$. Put

$$
A_{p, 4}=\overline{\mathbb{F}_{p}}\left[\left[X_{0}, X_{1}, \ldots, X_{4}\right]\right] /\left(X_{0}^{2}+\cdots+X_{4}^{2}\right) .
$$

Then the following statement holds.

(1) If $A$ is not regular, then

$$
e_{\mathrm{HK}}(A) \geq e_{\mathrm{HK}}\left(A_{p, 4}\right)=\frac{29 p^{2}+15}{24 p^{2}+12} .
$$

(2) The following conditions are equivalent:

(a) Equality holds in (1).

(b) $e_{\mathrm{HK}}(A)<\frac{5}{4}$.

(c) The completion of $A$ is isomorphic to $A_{p, 4}$.

Proof. Put $e=e(A)$, the multiplicity of $A$. We may assume that $A$ is complete with $e \geq 2$ and $k$ is infinite. In particular, $A$ is a homomorphic image of a Cohen-Macaulay local ring, and there exists a minimal reduction $J$ of $\mathfrak{m}$. Then $\mu_{A}\left(\mathfrak{m} / J^{*}\right) \leq e-1$ by Lemma 2.5. We first show that $e_{\mathrm{HK}}(A) \geq \frac{5}{4}$ if $e \geq 3$.

Claim 1. If $3 \leq e \leq 10$, then $e_{\mathrm{HK}}(A) \geq \frac{5}{4}$.

Putting $r=e-1$ and $s=2$ in Theorem 2.2, since $v_{2}=\frac{1}{2}$, we have

$$
e_{\mathrm{HK}}(A) \geq e\left\{v_{2}-\frac{(e-1) 1^{4}}{4 !}\right\}=\frac{(13-e) e}{24} \geq \frac{(13-3) \cdot 3}{24}=\frac{30}{24}
$$

as required.

Claim 2. If $11 \leq e \leq 29$, then $e_{\mathrm{HK}}(A) \geq \frac{737}{384}\left(>\frac{5}{4}\right)$. 
By Fact 2.4, we have $v_{3 / 2}=\frac{1-\beta_{4+1}}{2}=\frac{77}{384}$. Putting $r=e-1$ and $s=\frac{3}{2}$ in Theorem 2.2, we have

$$
e_{\mathrm{HK}}(A) \geq e\left\{v_{3 / 2}-\frac{e-1}{24} \cdot\left(\frac{1}{2}\right)^{4}\right\}=\frac{(78-e) e}{384} \geq \frac{11(78-11)}{384}=\frac{737}{384},
$$

as required.

Claim 3. If $e \geq 30$, then $e_{\mathrm{HK}}(A) \geq \frac{5}{4}$.

By Proposition 1.4, we have $e_{\mathrm{HK}}(A) \geq \frac{e}{4 !} \geq \frac{30}{24}$.

In the following, we assume that $k=\bar{k}$, char $k \neq 2$ and $e \geq 2$. To see (1), (2), we may assume that $e=2$ by the above argument. Then since $e_{\mathrm{HK}}(A)=2$ if $A$ is not $F$-rational, we may also assume that $A$ is $F$-rational and thus is a hypersurface. Thus $A$ can be written as the following form:

$$
A=k\left[\left[X_{0}, X_{1}, \ldots, X_{4}\right]\right] /\left(X_{0}^{2}-\varphi\left(X_{1}, X_{2}, X_{3}, X_{4}\right)\right) .
$$

If $A$ is isomorphic to $A_{p, 4}$, then by [10], it is known that $e_{\mathrm{HK}}(A)=\frac{29 p^{2}+15}{24 p^{2}+12}$. Suppose that $A$ is not isomorphic to $A_{p, 4}$. Then one can take a minimal numbers of generators $x, y, z, w, u$ of $\mathfrak{m}$ and one can define a function ord $: A \rightarrow \mathbb{Q} \cup\{\infty\}$ such that

$$
\operatorname{ord}(x)=\operatorname{ord}(y)=\operatorname{ord}(z)=\operatorname{ord}(z)=\frac{1}{2}, \quad \operatorname{ord}(u)=\frac{1}{3} .
$$

If we put $J=(y, z, w, u) A$ and $F_{n}=\{\alpha \in A: \operatorname{ord}(\alpha) \geq n\}$, then by a similar argument as in the proof of Proposition 3.9, we have

$$
l_{A}\left(\mathfrak{m}^{[q]} / J^{[q]}\right) \leq 2 \cdot l_{A}\left(A / J^{[q]}+F_{2 q / 3}\right) .
$$

Divided the both-side by $q^{d}$ and taking a limit $q \rightarrow \infty$, we get $e(A)-e_{\mathrm{HK}}(A) \leq 2 \cdot e(A) \cdot \operatorname{vol}\left\{(y, z, w, u) \in[0,1]^{4}: \frac{y}{2}+\frac{z}{2}+\frac{w}{2}+\frac{u}{3} \leq \frac{2}{3}\right\}$.

To calculate the volume in the right-hand side, we put

$$
F_{u}= \begin{cases}\frac{1}{6}\left(\frac{4}{3}-\frac{2}{3} u\right)^{3}-3 \cdot \frac{1}{6}\left(\frac{1}{3}-\frac{2}{3} u\right)^{3} & \left(0 \leq u \leq \frac{1}{2}\right) \\ \frac{1}{6}\left(\frac{4}{3}-\frac{2}{3} u\right)^{3} & \left(\frac{1}{2} \leq u \leq 1\right)\end{cases}
$$


Then one can easily calculate

$$
\text { the above volume }=\int_{0}^{1} F_{u} d u=\frac{237}{2^{4} 3^{4}} .
$$

It follows that

$$
e_{\mathrm{HK}}(A) \geq 2-4 \times \frac{237}{2^{4} 3^{4}}=\frac{411}{324}>\frac{5}{4}
$$

The following conjecture also holds if $\operatorname{dim} A \leq 4$.

ConjeCture 4.4. Under the same notation as in Conjecture 4.2, if $e(A) \geq 3$, then

$$
e_{\mathrm{HK}}(A) \geq 1+\frac{c_{d}+1}{d !}
$$

Discussion 4.5. Let $d \geq 2$ be an integer and fix a prime number $p \gg d$. Assume that Conjectures 4.2 and 4.4 are true. Also, assume that $s_{\mathrm{HK}}(p, d)<s_{\mathrm{HK}}(p, d-1)$ for all $d \geq 3$. Let $A=k\left[X_{0}, \ldots, X_{v}\right] / I$ be a $d$-dimensional homogeneous unmixed $k$-algebra with $\operatorname{deg} X_{i}=1$, and let $\mathfrak{m}$ be the unique homogeneous maximal ideal of $A$. Suppose that $k$ is an algebraically closed field of characteristic $p>0$. Then $e_{\mathrm{HK}}(A)=s_{\mathrm{HK}}(p, d)$ implies that $\widehat{A_{\mathfrak{m}}} \cong A_{p, d}$.

In fact, if $e_{\mathrm{HK}}(A)=s_{\mathrm{HK}}(p, d)$, then we may assume that $e_{\mathrm{HK}}(A)<$ $1+\frac{c_{d}+1}{d !}$. Thus $e\left(A_{\mathfrak{m}}\right)=2$ if Conjecture 4.4 is true. For any prime ideal $P A_{\mathfrak{m}}$ of $A_{\mathfrak{m}}$ such that $P \neq \mathfrak{m}$, we have $e_{\mathrm{HK}}\left(A_{P}\right) \leq e_{\mathrm{HK}}\left(A_{\mathfrak{m}}\right)=s_{\mathrm{HK}}(p, d)<$ $s_{\mathrm{HK}}(p, n)$, where $n=\operatorname{dim} A_{P}<d$. Since $A_{P}$ is also unmixed, it is regular. Thus $A_{\mathfrak{m}}$ has an isolated singularity. Hence $A$ is a non-degenerate quadric hypersurface In other words, $\widehat{A_{\mathfrak{m}}}$ is isomorphic to $A_{p, d}$.

\section{REFERENCES}

[1] R. O. Buchweitz and Q. Chen, Hilbert-Kunz functions of cubic curves and surfaces, J. Algebra, 197 (1997), 246-267.

[2] R. O. Buchweitz, Q. Chen and K. Pardue, Hilbert-Kunz functions, preprint.

[3] M. Blickle and F. Enescu, On rings with small Hilbert-Kunz multiplicity, Proc. Amer. Math. Soc., 132 (2004), 2505-2509.

[4] A. Conca, Hilbert-Kunz functions of monomials and binomial hypersurfaces, Manuscripta Math., 90 (1996), 287-300. 
[5] K. Eto and K. Yoshida, Notes on Hilbert-Kunz multiplicity of Rees algebras, Comm. Algebra, 31 (2003), 5943-5976.

[6] R. Fedder and K.-i. Watanabe, A characterization of F-regularity in terms of F-purity, Commutative algebra (Berkeley, CA, 1987), Math. Sci. Research Inst. Publ., vol. 15, Springer-Verlag, New York (1989), pp. 227-245.

[7] N. Fakhruddin and V. Trivedi, Hilbert-Kunz functions and multiplicities for full flag varieties and elliptic curves, J. Pure Appl. Algebra, 181 (2003), 23-52.

[8] S. Goto and Y. Nakamura, Multiplicity and tight closures of parameters, J. Algebra, 244 (2001), 302-311.

[9] D. Hanes, Notes on the Hilbert-Kunz function, Comm. Algebra, 30 (2002), 3789-3812.

[10] C. Han and P. Monsky, Some surprising Hilbert-Kunz functions, Math. Z., 214 (1993), 119-135.

[11] M. Hochster and C. Huneke, Tight closure, invariant theory, and the Briançon-Skoda theorem, J. Amer. Math. Soc., 3 (1990), 31-116.

[12] M. Hochster and C. Huneke, F-regularity, test elements, and smooth base change, Trans. Amer. Math. Soc., 346 (1994), 1-62.

[13] C. Huneke, Tight closure and its applications, American Mathematical Society, 1996.

[14] C. Huneke and Y. Yao, Unmixed local rings with minimal Hilbert-Kunz multiplicity are regular, Proc. Amer. Math. Soc., 130 (2002), 661-665.

[15] E. Kunz, Characterizations of regular local rings of characteristic p, Amer. J. Math., 41 (1969), 772-784.

[16] E. Kunz, On Noetherian rings of characteristic p, Amer. J. Math., 88 (1976), 999-1013.

[17] H. Matsumura, Commutative ring theory, Cambridge University Press, 1986.

[18] P. Monsky, The Hilbert-Kunz function, Math. Ann., 263 (1983), 43-49.

[19] P. Monsky, A personal letter from Monsky to K.-i. Watanabe.

[20] M. Nagata, Local rings, Interscience, 1962.

[21] D. Rees, A note on analytically unramified local rings, J. London Math. Soc., 36 (1961), 24-28.

[22] K.-i. Watanabe and K. Yoshida, Hilbert-Kunz multiplicity and an inequality between multiplicity and colength, J. Algebra., 230 (2000), 295-317.

[23] K.-i. Watanabe and K. Yoshida, Hilbert-Kunz multiplicity of two-dimensional local rings, Nagoya Math. J., 162 (2001), 87-110.

[24] K.-i. Watanabe and K. Yoshida, Hilbert-Kunz multiplicity, McKay correspondence and good ideals in two-dimensional rational singularities, Manuscripta Math., 104 (2001), 275-294. 
Kei-ichi Watanabe

Department of Mathematics

College of Humanities and Sciences

Nihon University

Setagaya-ku

Tokyo 156-0045

Japan

watanabe@math.chs.nihon-u.ac.jp

Ken-ichi Yoshida

Graduate School of Mathematics

Nagoya University

Chikusa-ku

Nagoya 464-8602

Japan

yoshida@math.nagoya-u.ac.jp 